\title{
THE CHALLENGES, UNCERTAINTIES AND OPPORTUNITIES OF BIOAEROSOL DISPERSION MODELLING FROM OPEN COMPOSTING FACILITIES
}

\author{
BEN WILLIAMS ${ }^{1}$, ENDA HAYES ${ }^{1}$, ZAHEER NASIR $^{2}$, CATHERINE ROLPH $^{3}$, SIMON JACKSON $^{4}$, \\ SHAGUN KHERA ${ }^{4}$, ALAN BENNETT ${ }^{5}$, TONI GLADDING ${ }^{3}$, GILLIAN DREW ${ }^{2} \&$ SEAN TYRREL $^{2}$ \\ ${ }^{1}$ Air Quality Management Resource Centre, University of the West of England, UK \\ ${ }^{2}$ School of Water, Energy and Environment, Cranfield University, UK \\ ${ }^{3}$ Integrated Waste Systems, The Open University, Milton Keynes, UK \\ ${ }^{4}$ School of Biomedical and Healthcare Sciences, Plymouth University, UK \\ ${ }^{5}$ Public Health England, Salisbury, UK
}

\begin{abstract}
Bioaerosols are ubiquitous organic particles that comprise viruses, bacteria and coarser fractions of organic matter. Known to adversely affect human health, the impact of bioaerosols on a population often manifests as outbreaks of illnesses such as Legionnaires Disease and Q fever, although the concentrations and environmental conditions in which these impacts occur are not well understood. Bioaerosol concentrations vary from source to source, but specific human activities such as water treatment, intensive agriculture and composting facilitate the generation of bioaerosol concentrations many times higher than natural background levels. Bioaerosols are not considered 'traditional' pollutants in the same way as $\mathrm{PM}_{10}, \mathrm{PM}_{2.5}$, and gases such as $\mathrm{NO}_{2}$, and consequently dispersion models do not include a bespoke method for their assessment. As identified in previous studies, priority areas for improving the robustness of these dispersion models include: 1) the development of bespoke monitoring studies designed to generate accurate modelling input data; 2 ) the publication of a robust emissions inventory; 3 ) a code of practice to provide guidelines for consistent bioaerosol modelling practices; and 4) a greater understanding of background bioaerosol emissions. The aim of this research project, funded by the Natural Environmental Research Council (NERC), is to address these key areas through a better understanding of the generation, concentration and potential dispersion of bioaerosols from intensive agricultural and biowaste facilities, using case studies developed at specific locations within the UK. The objective is to further refine existing bioaerosol monitoring and modelling guidelines to provide a more robust framework for regulating authorities and site operators. This contribution outlines the gaps that hinder robust dispersion modelling, and describes the on-site bioaerosol data collection methods used in the study, explaining how they might be used to close these gaps. Examples of bioaerosol dispersion modelled using ADMS 5 are presented and discussed.

Keywords: bioaerosols, ADMS, dispersion modelling, composting.
\end{abstract}

\section{INTRODUCTION}

Since the introduction of the Waste Framework Directive, 2008/96/EC, there has been a significant increase in the rate of recycled and composted waste. The Directive requires the UK and other Member States to recycle $50 \%$ of their household waste by 2020 . Comprehensive data collected for the UK since 2010 show that recycling rates for the UK as a whole are at $40.4 \%$. Variability exists between devolved administrations, with Wales recycling $44 \%$ of domestic waste and Scotland recycling 32.5\% [1].

The 2008/96/EC target specifically for biodegradable municipal waste sent to landfill is $35 \%$ of a Nation's 1997 baseline (considered to be the total tonnage sent to landfill in 1997). In the UK's case this is equivalent to a reduction from the baseline of 21,460 Ktonnes, to 7511 Ktonnes. 
In 2015 there were over 300 licensed composting facilities in the UK using a number of technologies including in-vessel, open, and closed composting systems. It is likely that their number will increase over the coming years as more waste is diverted from landfill.

Composting is the natural process of organic matter degradation by microorganisms. The more organic matter that is composted, the more micro-organisms will be present and consequently there is a potential for the release of a higher concentration of bioaerosols into the air. Bioaerosols, typically comprised of bacteria, fungi, and their components, are airborne microorganisms, released naturally into the air from the degradation of organic matter [2]. These ambient concentrations can be increased by anthropogenic activities, such as leaf blowing and the turning of composted garden waste. On an industrial scale, bioaerosols can be released from sewage plants during pre-treatment, aeration and sludge digestion [3], and from composting facilities during turning, screening and shredding activities [4].

Some bioaerosols are known to have adverse health effects, particularly for the immunecompromised. Brucellosis, Q Fever and Rift Valley fever are amongst the most well-known and are caused by exposure to Brucella spp, Coxiella burnettii and Phlebovirus respectively [5]. Due to the potential impact of bioaerosols on human health within the UK, the Environment Agency has determined that a site specific risk assessment will be required for any composting facility that is within $250 \mathrm{~m}$ of any sensitive receptors (such as homes), and they will need to demonstrate that bioaerosols are maintained at acceptable levels [6]. These levels, as measured by the AfOR standard protocol, are 300,1000 , and $500 \mathrm{cfu} \mathrm{m}^{-3}$ for gram-negative bacteria, total mesophilic bacteria, and Aspergillus fumigatus respectively. The sampling devices used in the current research project and the means by which their data may be used in subsequent modelling activities are set out below.

Some progress has been made in characterising emissions from composting facilities, however relatively little headway has been made regarding the linked research questions of: understanding exposure of the general public to bioaerosols; putting process-based exposures into the context of background exposure to natural bioaerosols (or other anthropogenic sources); and quantifying health risk and setting health-based standards. A critical limiting factor in all of these areas is the lack of advanced microbiological methods (sampling, analytical, interpretative) to quantify and qualify bioaerosol emissions and dispersion.

This research project aims to develop new methodologies capable of characterising and quantifying emissions of bioaerosol into the air. Our objectives include: i) developing new methods to size fractionate endotoxin and elucidate its structural features; ii) developing a novel biosensor for rapid detection of endotoxin, other inflammatory agents and cells (live/dead); iii) using the Wideband Integrated Bioaerosol Sampler (WIBS) real-time bioaerosol sensor to understand the emission and dispersion of bioaerosol; iv) characterising industry-specific bioaerosol emissions at composting and farm sites; v) detecting microbial pathogens at biowaste and intensive agricultural facilities; and vi) generating improved exposure assessments of biowaste/intensive agricultural facilities using dispersion modelling and Openair resources.

\section{BIOAEROSOL MODELLING}

Spatially, air pollution can be modelled on local, national, and global levels, depending on the pollutant. Emissions from composting facilities are not expected to disperse in significant concentrations beyond the near vicinity and as such, short-range modelling is appropriate. Models for assessing local sources generally focus on one of the following five standard categories: 
- Point

- Area

- Line

- Volume

- Jet

Models focusing on point sources are generally used to model pollutants from stacks and other discharge points. Models focusing on area and volume sources are used to assess emissions from a designated area. Other models focusing on line sources are used to assess emissions from roads, whilst models considering jet emissions are used to assess the release of a pollutant from mechanically ventilated areas or where the discharged pollutant has been discharged at speed. Numerous modelling packages exist for the assessment of these different approaches and each one is unique in its output. As noted by Douglas [7] and CERC [8], the ADMS models are most appropriate for assessing bioaerosol dispersion from composting facilities, but AERMOD is also suitable. The challenges and uncertainties set out below relate to ADMS, however many will also be relevant to other models.

\section{ADDRESSING THE CHALLENGES AND UNCERTAINTIES ASSOCIATED WITH BIOAEROSOL DISPERSION MODELLING}

The composting of green waste involves a number of materials handling processes from which bioaerosols are generated. Waste is initially brought on-site and disposed of at a designated area before being shredded and placed in windrows. The windrows are then frequently turned in order to introduce fresh oxygen into the material allowing the aerobic bacteria to continue the degradation process. Material from wind rows are then taken to be screened and subsequently sold.

The primary challenges and uncertainties associated with bioaerosol monitoring, are as a result of a lack of data and the difficulty in deriving source term data from different site based operations. As set out within Cambridge Environmental Research Consultants (CERC) Atmospheric Dispersion Modelling Liaison Committee (ADMLC) report the primary limitations relate to source definition, meteorology, emission rates, observation uncertainty and general model performance. This project aims to address a number of these through the monitoring, modelling and analysis of data at a composting facility in England, known herein as Site A. The strategy for addressing them is set out below.

In this study, data have been collected from Site A, a facility which composts 25,000 tonnes of green waste a year. The site is divided into two distinct processing areas; firstly, waste is brought on to site and tipped onto the ground within a small sector of the windrow hardstanding area before being shredded and arranged into windrows. Secondly, mature compost from aged windrows is taken to the western sector of the site for screening into saleable compost.

\subsubsection{Source definition}

Defining the source of pollutants on site is the first step in modelling its release on a site. Sources of pollutants on site can be modelled individually or treated as one contiguous source. This will depend on the quality of data available for a given site. As shown in Fig. 1, the layout of site operations are known in detail at Site A. Each individual compost windrow is defined within the model instead of being considered as one source. This is to account for the fact that each wind-row will be at a different maturation phase to the next and will be turned and screened at different times. 


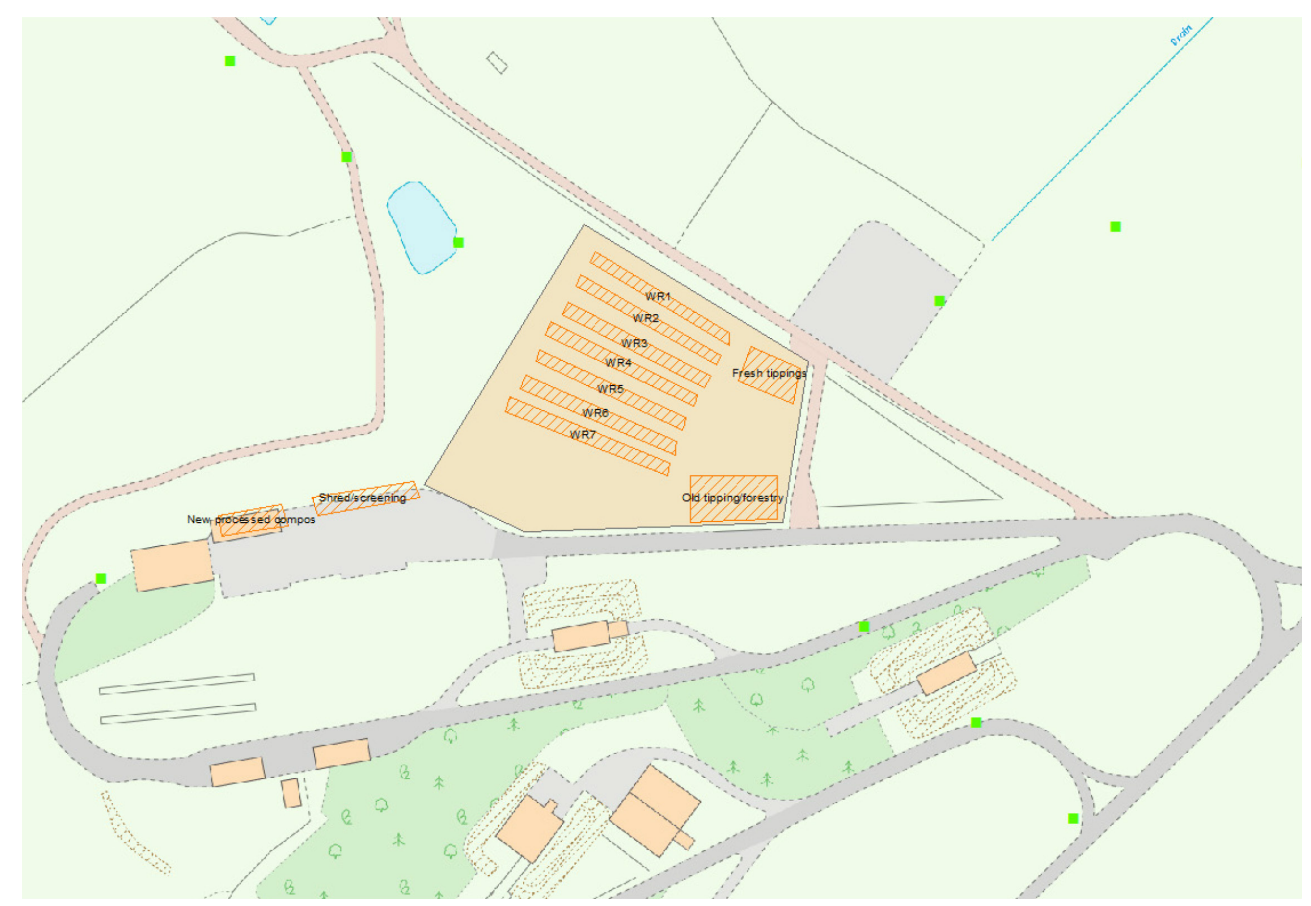

Figure 1: Site A layout.

In addition to the separation of sources on site, their height is a key parameter that requires defining within the model. Agitation will occur at different heights within a windrow, depending on the equipment used, however it is very difficult to accurately assess the height at which agitation takes place. Despite this, Douglas [7] and CERC [8] suggest that for sites where data are not available, a height of 2.65 metres is used.

Time-varying emission factors allow a model to incorporate changes in emissions across the day from various on-site activities into the model to provide a more accurate picture of pollutant generation and dispersion. A site activity log can also corroborate the data gathered from the SIBS to reinforce the inclusion of specific time-varying emission factors.

\subsubsection{Emission rates}

Standard models do not consider bioaerosols as a distinct pollutant within their algorithms and as such the common practice is to use particulate matter, be it TSP or $\mathrm{PM}_{10}$ as a proxy [9]. In doing so bioaerosols are assumed within the model to behave in similar ways to particulate matter.

Exit velocity and temperature are key parameters when modelling emissions. However, Douglas [7] notes that there are no reliable exit velocities of bioaerosol emissions from composting windrows, and an exit velocity of $2.95 \mathrm{~ms}^{-1}$ is considered appropriate. Temperature will also affect plume rise in the model and CERC [8] considered modelling with a predetermined temperature of $29^{\circ} \mathrm{C}$. Both exit velocity and temperatures set out here were considered by Douglas to be an average of the efflux parameters, falling between higher velocities and temperatures during the turning and mixing, and the relatively lower temperatures and exit velocities from non-agitated compost [7]. 


\subsubsection{Meteorological data collection}

When identifying met data for use at a particular site, the ideal scenario would be robust, reliable data collected on the site itself. Where this is not possible, data can be obtained from nearby meteorological stations, processed an input into ADMS. Meteorological data are available from the British Atmospheric Data Centre (BADC), the Natural Environment Research Council's (NERC) Designated Data Centre for the Atmospheric Sciences through the Centre for Environmental Data Analysis [10]. Without accurate met data, models will not be able to assess the dispersion of pollutants into the surrounding area. Within ADMS, data on temperature, rainfall, humidity, wind speed, wind direction and cloud cover are required. Furthermore, if met data are used from stations hundreds of kilometres away then the met data will not be reflective of local conditions and impacts not properly assessed.

\subsubsection{Other considerations}

When considering the modelling of pollutants, it is important to recognise that even if source terms are identical within distinct models, the modelled concentration will likely differ between them [8]. As noted within CERC [8], the SCAIL Agriculture update report [11] has several datasets that may be used to validate models, however their accessibility is limited. This current study aims to verify the modelled concentrations against data collected using the SIBS.

Time-varying emission factors are likely to play an important role in assessing the extent of dispersion at Site A and subsequently elsewhere. As shown in Fig. 2, the difference between a model run with identical parameters, except an adjustment in an emission factor at $11 \mathrm{am}$, result in a different dispersion profile. With such an important change to the profile, additional receptors may be considered at risk and thus further scrutiny at those locations may be required.

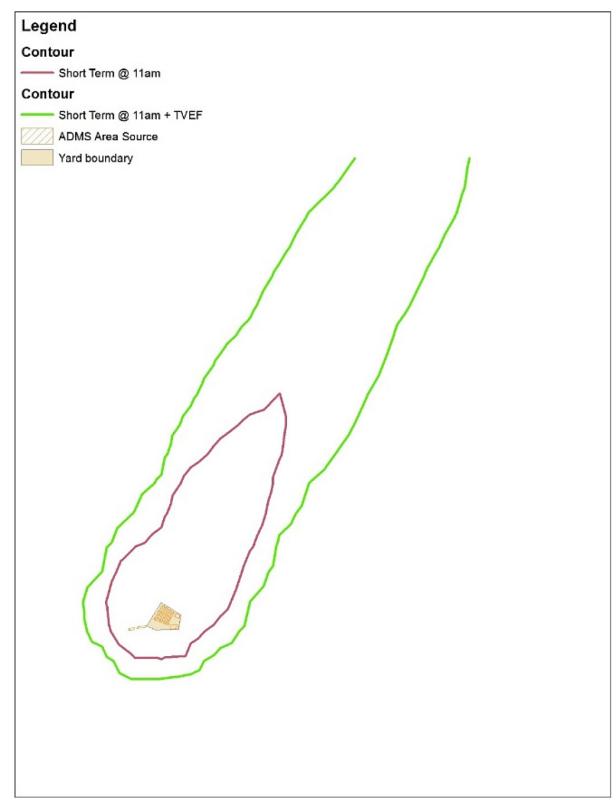

Figure 2: Short-term model of bioaerosol dispersion with and without time-varying emission factors. 
As set out above, there are several gaps in data that need to be addressed in order to improve the quality of bioaerosol modelling activities. The following section sets out the monitoring methods used to address the data gaps identified above and subsequently summarised in Table 1.

\subsection{Bioaerosol monitoring}

Bioaerosol monitoring approaches are set out within the AfOR protocol [6], produced by the Environment Agency. Within this protocol, the appropriate samplers to be used are set out and the medium upon which samples are collected are presented. The protocol also sets out the appropriate monitoring locations and the timeframes over which samples should be collected. The measurement of bioaerosols is regularly undertaken with the aim of identifying the presence of viable organisms, quantified as colony forming units per cubic metre $\left(\mathrm{CFU} \mathrm{m}{ }^{-3}\right)$. Alternatively, real-time bioaerosol monitors may be able to discern specific bioaerosol components and concentrations at different wavelengths.

The Institute of Occupational Medicine's (IOM) personal sampler has been used regularly for bioaerosol sampling [4], [12], [13]. Compared with other bioaerosol samplers, they are inexpensive, lightweight and easily deployable in many locations. The IOM samplers typically run for 30 minutes at $2.2 \mathrm{~L} / \mathrm{min}$, with air drawn through a $0.8 \mathrm{~m}$ pore size polycarbonate filter [12]. The benefits of these samplers are that a physical sample is collected allowing further analysis to be undertaken elsewhere. The IOM allows for an understanding of bioaerosol concentrations over time, and coupled with other sampling methods may provide valuable source term data.

Multi-stage Andersen impactors are frequently used to assess bioaerosol concentrations. The Andersen samplers are often fitted with a hemispherical baffle, and micro-organisms are collected onto either nutrient agar or tryptone soya agar (TSA) and malt extract agar (MEA) [14]. Andersen samplers are valuable sampling tools, particularly when using a multi-stage sampler as these will allow the collection and subsequent analysis of bioaerosols at different particle sizes. Coupled with IOM samplers, the multi-stage Andersen allows for the determination of specific bioaerosols and subsequently, through the analysis of each stage, their predominant particle size. This will facilitate a refinement of particle size data used in the model.

The Osiris particulate monitor is traditionally used as a screening tool for assessing particulate matter on and around site boundaries. The Osiris will continuously determine the concentration of Total suspended particulates (TSP), $\mathrm{PM}_{10}, \mathrm{PM}_{2.5}$ and $\mathrm{PM}_{1}$ particles with a resolution of $0.1 \mu \mathrm{g} / \mathrm{m}^{3}$. The Osiris will complement the data gathered by the Andersen sampler and provide information of particle size of emissions at and around the composting facility.

Table 1: Model data gaps and solutions.

\begin{tabular}{|l|c|c|c|c|c|}
\hline Model data gaps & $\begin{array}{l}\text { Bioaerosol } \\
\text { composition }\end{array}$ & $\begin{array}{l}\text { Emission } \\
\text { rates }\end{array}$ & $\begin{array}{l}\text { Particle } \\
\text { size }\end{array}$ & $\begin{array}{l}\text { Time varying } \\
\text { emissions }\end{array}$ & $\begin{array}{l}\text { Model } \\
\text { validation }\end{array}$ \\
\hline IOM & $\mathrm{x}$ & $\mathrm{x}$ & & & $\mathrm{x}$ \\
\hline Coriolis & $\mathrm{x}$ & $\mathrm{x}$ & & & \\
\hline Multi-stage Andersen & $\mathrm{x}$ & $\mathrm{x}$ & $\mathrm{x}$ & & \\
\hline Osiris & & $\mathrm{x}$ & $\mathrm{x}$ & $\mathrm{x}$ & $\mathrm{x}$ \\
\hline SIBS & $\mathrm{x}$ & $\mathrm{x}$ & $\mathrm{x}$ & $\mathrm{x}$ & $\mathrm{x}$ \\
\hline
\end{tabular}


Analysis of bioaerosol composition is also undertaken through the use of high volume samplers such as the Coriolis [15] quantification of bioaerosols collected using the Coriolis is usually accomplished by flow cytometry and chemiluminescence sandwich immunoassays [15]. Data from the Coriolis can be used to identify the types of bioaerosols present in air and as such can provide further information on the dominant organic components present at each sampling site.

The Spectral Intensity Bioaerosol Sensor (SIBS) measures particle size and the fluorescence intensity of particles across 16 wavelength intensities (from $288 \mathrm{~nm}$ to $735 \mathrm{~nm}$ ) to detect bioaerosols. The instrument allows for the determination of particle size, shape, asphericity and the measurement of fluorescence, which may indicate the presence of bioaerosols or other organic components at a high temporal resolution. Further technical details of the SIBS are set out in [16]. High resolution data from the SIBS may provide an understanding of site activities and thus contribute directly to the time-varying emission factors used in the modelling process and be used to determine emission rates from specific site activities. SIBS data can also be used to verify and validate model outputs.

\section{OPPORTUNITIES}

There are numerous challenges and uncertainties inherent in this research project, but at the same time opportunities abound. A bespoke, in depth monitoring study is currently underway at Site A and similar studies will also be conducted at a broiler farm and at a background site in order to assess bioaerosols at different operations.

The diversity of monitoring data gathered from these sites will also allow for a comparison of bioaerosols present in different size fractions (and whether there are differences between fractions), a screen of the types of bioaerosols present in the samples and an analysis of the potential health impacts of those identified.

Through the use of the SIBS and IOM samplers a real-time profile of bioaerosol dispersion at and around each site will be gathered. Furthermore, emission factors for shredding, turning and screening activities will be determined which will aid in improving the model's accuracy.

\section{DISCUSSION}

The European Waste Directive's drive to divert waste from landfill to recycling and composting facilities has resulted in the increase of such facilities across the UK over the last decade. As more waste is diverted from landfill these numbers will likely rise further and consequently there will be an increase in the number of people living within close proximity to such a facility. Understanding the health impacts of bioaerosols, their concentration and the extent of dispersion around these facilities will therefore become increasingly important.

This contribution has set out the gaps in data required for robust bioaerosol modelling and the means being undertaken to fill these. Bioaerosol composition will fluctuate depending on site activities, and the range of monitoring devices used in this study will be able to identify the peaks in bioaerosol release and the associated activities. Both the IOM and Andersen provide valuable information for the modelling of bioaerosols. At Site A, the IOM will determine the source emissions while the multi-stage Andersen sampler will ascertain the general particle size of the bioaerosols and the appropriate proxy particle size selected within the model. These standard methodologies set out within AfOR are considered to be spot samplers, providing a sample at a given location for a given averaged timeframe. Spot samplers typically lack high time resolution and require frequent change-overs. This limits a detailed understanding of short-term site activities such as turning and screening and, without significant investment, makes the interpretation of diurnal and seasonal variability a challenge. The use of the SIBS aims to address this time resolution challenge. These data will 
be crucial for the accurate definition of time varying emission factors on site, a factor which could have a significant impact on the extent of bioaerosol dispersion and likely exposure. A challenge remains in unpicking the meaning of the fluorescence data, but once this has been achieved, detailed information on specific bioaerosols will be available for interpretation. A potential research avenue will be to build up a database of fluorescence measurements from known bioaerosols against which on-site data can be compared.

Determining activity-specific emission factors will require samplers to be introduced close to the point of release and this will present a technical challenge, however the data gained from such activities will certainly push the state of knowledge further in this field. Applying the methodology to measure bioaerosol emissions from broiler sheds will also create state of the art knowledge on bioaerosol dispersion.

The ultimate aim of this work is to contribute and update regulatory guidance and best practice documents so that site operators and regulators are clear on the relative risks of composting in England.

\section{CONCLUSION}

This contribution has set out the challenges, uncertainties and opportunities associated with bioaerosol modelling at composting facilities. Key challenges include the refinement of source term data, acquiring robust meteorological data, and understanding the temporal variability in emissions on site. The opportunities presented are clear and include the potential to provide state of the art knowledge on bioaerosol measurement and dispersion profiles around composting facilities, and to transpose methodologies learned at Site A on to other sites in which bioaerosols are a concern.

Finally, the opportunity to update guidance documents based on the findings of this research project and to use the data from this to aid in the modelling of bioaerosols at all composting facilities nationwide is key to the project's success.

\section{ACKNOWLEDGEMENT}

This work was funded by the Natural Environmental Research Council (NERC) (reference number: NE/M011747/1).

\section{REFERENCES}

[1] Department for Environment Food and Rural Affairs, UK Statistics on Waste, 2016. https://www.gov.uk/government/uploads/system/uploads/attachment data/file/59304 0/UK_statsonwaste statsnotice Dec2016_FINALv2_2.pdf. Accessed on: 26 Feb. 2017.

[2] Cox, C.S. \& Wathes, C.M., Bioaerosols in the environment. Bioaerosols Handbook, eds C.S. Cox \& C.M. Wathes, CRC Press: Boca Raton, FL, 1995.

[3] Li, J., Zhou, L., Zhang, X., Xu, C. \& Dong, L., Bioaerosol emissions and detection of airborne antibiotic resistance genes from a wastewater treatment plant. Atmospheric Environment, 124, pp. 404-412, 2016.

[4] Taha, M.P.M., Drew, G.H., Longhurst, P.J., Smith, R. \& Pollard, S.J.T., Bioaerosol releases from compost facilities: Evaluating passive and active source terms at a green waste facility for improved risk assessments. Atmospheric Environment, 40(6), pp. 1159-1169, 2006.

[5] Kanoute, Y.B., Gragnon, G.G., Schindler, C., Bonfoh, B. \& Schelling, E., Epidemiology of Brucellosis, Q Fever and Rift Valley Fever at the human and livestock interface in northern Cote d'Ivoire. Acta Tropica, 165, pp. 66-75, 2017. 
[6] AfOR, The State of Composting and Biological Waste Treatment in the UK 2006/07, Association for Organics Recycling: Northamptonshire, Wellingborough, 2008.

[7] Douglas, P., Bioaerosol emissions from open windrow composting facilities: Emission characterisation and dispersion modelling improvements, $\mathrm{PhD}$ thesis, University of Cranfield, 2013.

[8] CERC, A review of the limitations and uncertainties of modelling pollutant dispersion from non-point sources. Contract report for the Atmospheric Dispersion Modelling Liaison Committee, 2015.

[9] Williams, M., et al., Bioaerosols and odour emissions from composting facilities. Final Report, Defra Project WR 1121, 2013.

[10] Centre for Environmental Data Analysis, MIDAS UK Hourly Weather Observation Data. http://catalogue.ceda.ac.uk/uuid/916ac4bbc46f7685ae9a5e10451bae7c

[11] Hill, R., et al., SCAIL Agriculture Update. Sniffer ER26 Final Report, 2014.

[12] Pankhurst, L.J., et al., Temporal and spatial changes in the microbial bioaerosol communities in green-waste composting. Federation of European Microbiology Societies Microbiology Ecology, 79, pp. 229-239, 2012.

[13] Taha, M.P.M., Drew, G.H., Tamer Vestlund, A., Aldred, D., Longhurst, P.J. \& Pollard, S.J.T., Enumerating actinomycetes in compost bioaerosols at source - use of soil compost agar to address plate 'masking'. Atmospheric Environment, 40(22), pp. 4759-4765, 2007.

[14] Environment Agency, Review of methods to measure bioaerosols from composting sites. Better regulation Science Programme, Science Report: SC040021/SR3, 2009.

[15] Langer, V., Hartmann, G., Niessner, R. \& Seidel, M., Rapid quantification of bioaerosols containing L. pneumophila by Coriolis ${ }^{\circledR} \mu$ air sampler and chemiluminescence antibody microarrays. Journal of Aerosol Science, 48, pp. 46-55, 2012.

[16] Nasir, Z.A., et al., under consideration in Environmental Research Letters. 